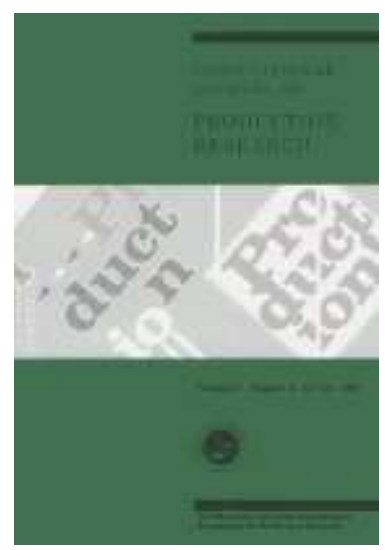

\title{
(19th ICPR) Production planning model with simultaneous production of spare parts
}

\begin{tabular}{|r|l|}
\hline Journal: & International Journal of Production Research \\
\hline Manuscript ID: & TPRS-2008-IJPR-0264.R2 \\
\hline Manuscript Type: & Original Manuscript \\
\hline Author: & 07-Nov-2008 \\
\hline Complete List of Authors: & $\begin{array}{l}\text { Cyplik, Piotr; Poznan University of Technology, Institute of } \\
\text { Management Engineering } \\
\text { Hadas, Lukasz; Poznan University of Technology, Institute of } \\
\text { Management Engineering } \\
\text { Fertsch, Marek; Poznan University of Technology, Institute of } \\
\text { Management Engineering }\end{array}$ \\
\hline Keywords: & PRODUCTION PLANNING, INVENTORY MANAGEMENT \\
\hline Keywords (user): & DECOUPLING POINT, SPARE PARTS \\
\hline $\begin{array}{l}\text { Note: The following files were submitted by the author for peer review, but cannot be converted } \\
\text { to PDF. You must view these files (e.g. movies) online. }\end{array}$ \\
\hline \begin{tabular}{l} 
TPRS-2008-IJPR-0264.R2 final.doc \\
\hline
\end{tabular}
\end{tabular}

\section{scholarONE" \\ Manuscript Central}




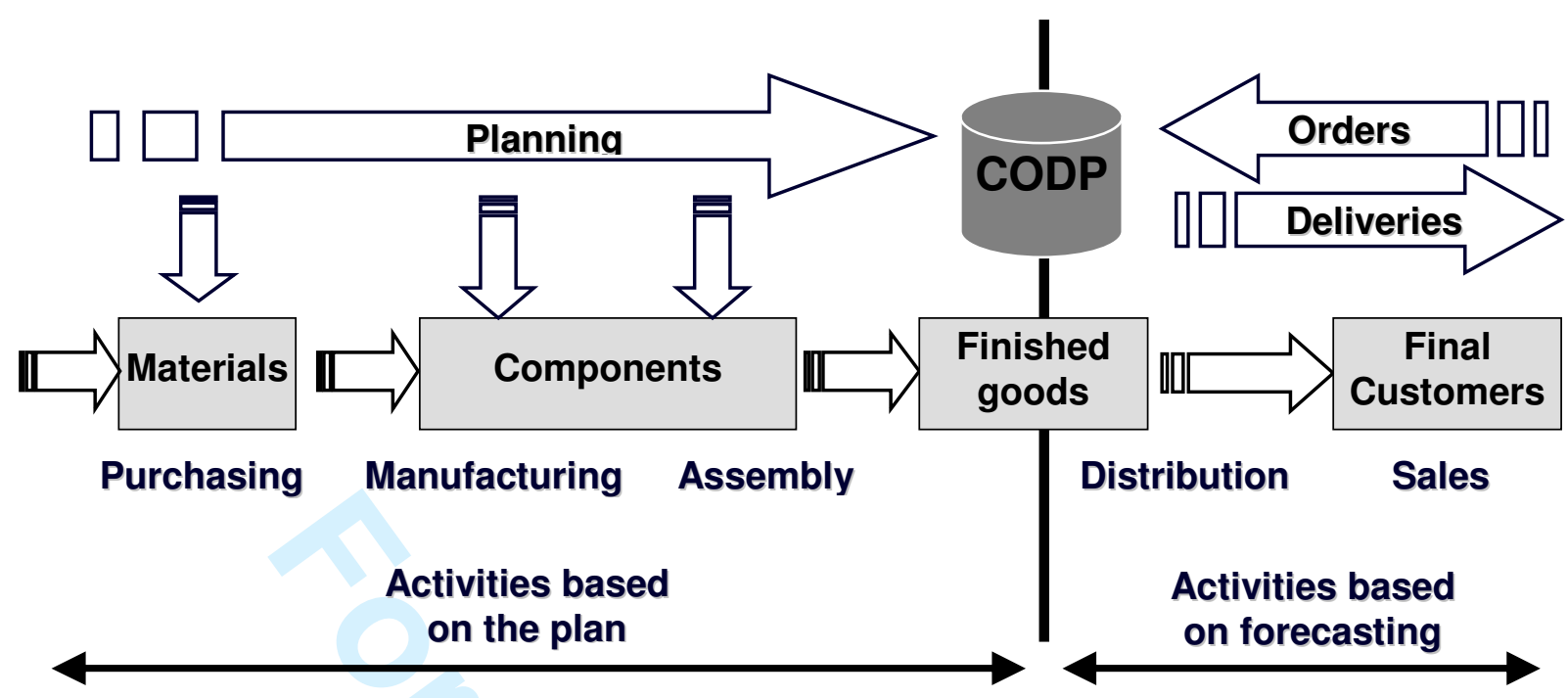

Figure 1. An example of CODP location

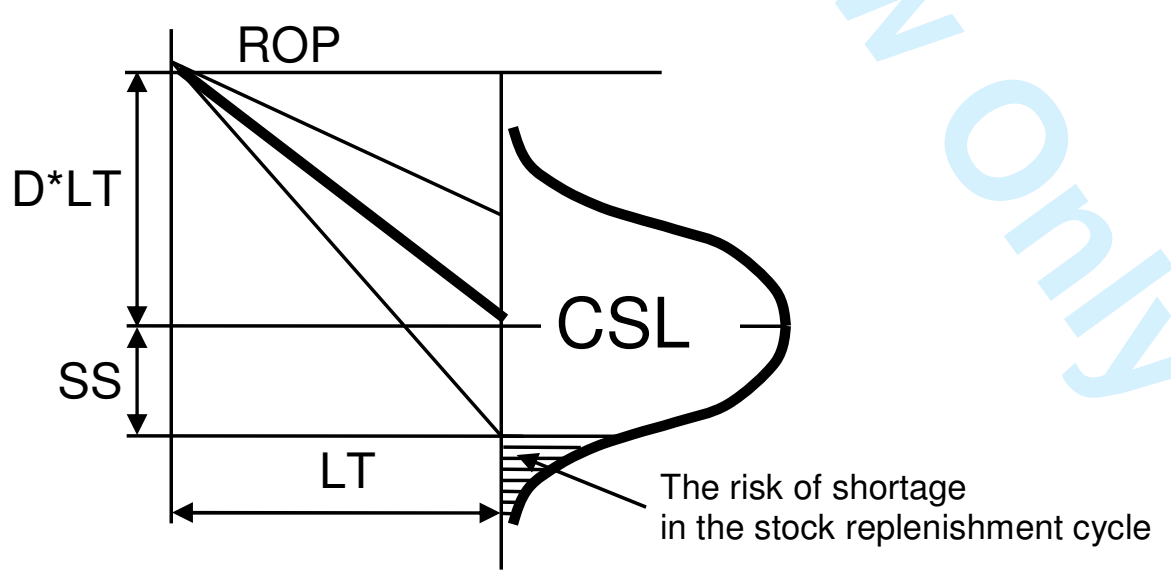

D - demand,

$\mathrm{LT}$ - replenishment lead time,

ROP - the level of re-order point,

SS - safety stock.

CSL - Cycle-Service Level.

Figure 2. Graphic presentation of Cycle-Service Level. 
Table 1. Suggested $\mathrm{x}_{1}$ and $\mathrm{x}_{2}$ parameters value in 1-2-3 analysis.

\begin{tabular}{|c|c|c|}
\hline Group & \multicolumn{2}{|c|}{ Scope } \\
\hline Group 1 & \multirow{3}{*}{\multicolumn{2}{|c|}{$\begin{array}{c}\text { below } \mathrm{x}_{1} \\
\text { from } \mathrm{x}_{1} \text { to } \mathrm{x}_{2} \\
\text { more than } \mathrm{x}_{2}\end{array}$}} \\
\hline Group 2 & & \\
\hline Group 3 & & \\
\hline Parameter & $\begin{array}{c}\text { Suggested } \\
\text { parameter value } \\
\text { for low frequency } \\
\text { issues. }\end{array}$ & $\begin{array}{c}\text { Suggested } \\
\text { parameter value } \\
\text { for high frequency } \\
\text { issues. }\end{array}$ \\
\hline $\mathrm{x}_{1}$ & $1-4$ & $1-7$ \\
\hline $\mathrm{x}_{2}$ & $1-3$ & $1-4$ \\
\hline
\end{tabular}

Table 2. Methods of covering the requirement for spare parts assigned to particular categories according to $\mathrm{ABC}, 123$ and CVA analyses

\begin{tabular}{|c|c|c|c|c|}
\hline $\begin{array}{c}\mathrm{CVA} \\
/ \mathrm{ABC}+123\end{array}$ & VIP & IP & MIP & LIP \\
\hline A1 & $\begin{array}{l}\text { "re-order } \\
\text { point" }\end{array}$ & $\begin{array}{l}\text { "re-order } \\
\text { point" }\end{array}$ & $\begin{array}{l}\text { "re-order } \\
\text { point" }\end{array}$ & $\begin{array}{l}\text { "re-order } \\
\text { point" }\end{array}$ \\
\hline A2 & $\begin{array}{l}\text { "re-order } \\
\text { point" }\end{array}$ & $\begin{array}{l}\text { "re-order } \\
\text { point" }\end{array}$ & $\begin{array}{l}\text { "re-order } \\
\text { point" }\end{array}$ & $\begin{array}{l}\text { "re-order } \\
\text { point" }\end{array}$ \\
\hline A3 & "lot for lot" & "lot for lot" & "to order" & "to order" \\
\hline B1 & $\begin{array}{l}\text { "re-order } \\
\text { point" }\end{array}$ & $\begin{array}{l}\text { "re-order } \\
\text { point" }\end{array}$ & $\begin{array}{c}\text { "cycle } \\
\text { review" }\end{array}$ & $\begin{array}{l}\text { "cycle } \\
\text { review" }\end{array}$ \\
\hline B2 & $\begin{array}{c}\text { "re-order } \\
\text { point" }\end{array}$ & $\begin{array}{l}\text { "cycle } \\
\text { review" }\end{array}$ & $\begin{array}{l}\text { "cycle } \\
\text { review" }\end{array}$ & $\begin{array}{l}\text { "cycle } \\
\text { review" }\end{array}$ \\
\hline B3 & "lot for lot" & "lot for lot" & "to order" & "to order" \\
\hline $\mathrm{C} 1$ & $\begin{array}{l}\text { "re-order } \\
\text { point" }\end{array}$ & $\begin{array}{l}\text { "re-order } \\
\text { point" }\end{array}$ & $\begin{array}{c}\text { "cycle } \\
\text { review" }\end{array}$ & $\begin{array}{l}\text { "cycle } \\
\text { review" }\end{array}$ \\
\hline $\mathrm{C} 2$ & "lot for lot" & $\begin{array}{l}\text { "cycle } \\
\text { review" }\end{array}$ & $\begin{array}{l}\text { "cycle } \\
\text { review" }\end{array}$ & $\begin{array}{l}\text { "cycle } \\
\text { review" }\end{array}$ \\
\hline C3 & "lot for lot" & "lot for lot" & "to order" & "to order" \\
\hline $\mathrm{D}$ & "lot for lot" & "lot for lot" & "to order" & "to order" \\
\hline
\end{tabular}


Table 3. The method of the best covering the requirement for spare parts assigned to particular categories according to $\mathrm{ABC}, 123$ and CVA analyses - table for the Expert

\begin{tabular}{ccccc}
\hline $\begin{array}{c}\text { Covering the requirement / } \\
\text { Spare parts classification }\end{array}$ & $\begin{array}{c}\text { "re-order } \\
\text { point" }\end{array}$ & $\begin{array}{c}\text { "cycle } \\
\text { review" }\end{array}$ & "lot for lot" & "to order" \\
\hline A1/VIP & $\mathrm{X}$ & & & \\
A1/IP & $\mathrm{X}$ & & & \\
A1/MIP & $\mathrm{X}$ & & & \\
A1/LIP & $\mathrm{X}$ & & & \\
$\ldots$ & $\ldots$ & $\ldots$ & $\ldots$ & $\ldots$ \\
\hline
\end{tabular}


Table 4. Results of experts' opinions research (inventory management managers) for all groups

\begin{tabular}{|c|c|c|c|c|}
\hline $\begin{array}{l}\text { Covering the requirement / } \\
\text { Spare parts classification }\end{array}$ & $\begin{array}{l}\text { "re-order } \\
\text { point" }\end{array}$ & $\begin{array}{l}\text { "cycle } \\
\text { review" }\end{array}$ & "lot for lot" & "to order" \\
\hline A1/VIP & $18^{*}$ & 1 & 1 & 0 \\
\hline A1/IP & 19 & 0 & 1 & 0 \\
\hline A1/MIP & 17 & 1 & 1 & 1 \\
\hline A1/LIP & 17 & 0 & 2 & 1 \\
\hline A2/VIP & 18 & 2 & 0 & 0 \\
\hline $\mathrm{A} 2 / \mathrm{IP}$ & 17 & 1 & 1 & 1 \\
\hline A2/MIP & 17 & 2 & 1 & 0 \\
\hline A2/LIP & 17 & 2 & 1 & 0 \\
\hline A3/VIP & 2 & 0 & 16 & 2 \\
\hline A3/IP & 1 & 0 & 17 & 2 \\
\hline A3/MIP & 2 & 1 & 1 & 16 \\
\hline A3/LIP & 1 & 1 & 0 & 18 \\
\hline B1/VIP & 19 & 0 & 1 & 0 \\
\hline B1/IP & 17 & 1 & 1 & 1 \\
\hline B1/MIP & 2 & 16 & 1 & 1 \\
\hline B1/LIP & 2 & 15 & 2 & 1 \\
\hline B2/VIP & 17 & 1 & 1 & 1 \\
\hline B2/IP & 1 & 16 & 2 & 1 \\
\hline B2/MIP & 2 & 15 & 2 & 1 \\
\hline B2/LIP & 2 & 15 & 1 & 2 \\
\hline B3/VIP & 1 & 1 & 16 & 2 \\
\hline B3/IP & 1 & 1 & 17 & 1 \\
\hline B3/MIP & 2 & 1 & 1 & 16 \\
\hline B3/LIP & 1 & 1 & 1 & 17 \\
\hline $\mathrm{C} 1 / \mathrm{VIP}$ & 18 & 1 & 1 & 0 \\
\hline $\mathrm{C} 1 / \mathrm{IP}$ & 17 & 1 & 1 & 1 \\
\hline C1/MIP & 1 & 17 & 1 & 1 \\
\hline C1/LIP & 1 & 16 & 2 & 1 \\
\hline C2/VIP & 1 & 1 & 16 & 2 \\
\hline $\mathrm{C} 2 / \mathrm{IP}$ & 1 & 17 & 1 & 1 \\
\hline $\mathrm{C} 2 / \mathrm{MIP}$ & 2 & 16 & 1 & 1 \\
\hline C2/LIP & 1 & 16 & 2 & 1 \\
\hline C3/VIP & 1 & 0 & 17 & 2 \\
\hline C3/IP & 1 & 1 & 16 & 2 \\
\hline C3/MIP & 0 & 0 & 4 & 16 \\
\hline C3/LIP & 0 & 0 & 2 & 18 \\
\hline D/VIP & 1 & 0 & 18 & 1 \\
\hline
\end{tabular}

* The numbers in above table mean the quantity of answers given by experts for particular categories. 
Table 5. Relative classification dispersion rates for individual groups ABC/123/CVA

\begin{tabular}{ccccc}
\hline $\begin{array}{c}\text { CVA } \\
\text { IABC+123 }\end{array}$ & VIP & IP & MIP & LIP \\
\hline A1 & 0,247 & 0,127 & 0,360 & 0,353 \\
A2 & 0,240 & 0,360 & 0,353 & 0,353 \\
A3 & 0,453 & 0,353 & 0,460 & 0,247 \\
B1 & 0,127 & 0,360 & 0,460 & 0,553 \\
B2 & 0,360 & 0,460 & 0,553 & 0,553 \\
B3 & 0,460 & 0,360 & 0,460 & 0,360 \\
C1 & 0,247 & 0,360 & 0,360 & 0,460 \\
C2 & 0,460 & 0,360 & 0,460 & 0,460 \\
C3 & 0,353 & 0,460 & 0,427 & 0,240 \\
D & 0,247 & 0,353 & 0,340 & 0,240 \\
\hline
\end{tabular}

Table 6: Suggested values of customer service level (Cycle-Service Level) in terms of probability for spare parts assigned to particular categories according to CVA and $\mathrm{ABC}$ analyses

\begin{tabular}{ccccc}
\hline $\begin{array}{c}\text { CVA } \\
\text { IABC }\end{array}$ & VIP & IP & MIP & LIP \\
\hline A & $90 \%-99,99 \%$ & $75 \%-98 \%$ & $75 \%-98 \%$ & $75 \%-98 \%$ \\
B & $90 \%-99,99 \%$ & $75 \%-98 \%$ & $60 \%-80 \%$ & $50 \%-65 \%$ \\
C & $90 \%-99,99 \%$ & $75 \%-98 \%$ & $60 \%-80 \%$ & $50 \%$ \\
D & $90 \%-99,99 \%$ & $60 \%-80 \%$ & $50 \%$ & $50 \%$ \\
\hline
\end{tabular}

